Pacific Journal of Mathematics

LINEAR DIFFERENTIAL EQUATIONS ON CONES IN BANACH 


\title{
LINEAR DIFFERENTIAL EQUATIONS ON CONES IN BANACH SPACES
}

\author{
Charles V. Coffman
}

1. In [1], Hartman and Wintner show that if $A(t)$ is an $n \times n$ matrix of nonnegative, continuous functions, defined on the interval $[0, \infty)$ then the differential equation

$$
x^{\prime}=-A(t) x
$$

has at least one nontrivial solution $x(t)=\left(x_{1}(t), \cdots, x_{n}(t)\right)$ satisfying

$$
x(t) \geqq 0, \quad-x^{\prime}(t) \geqq 0 \text { for } 0<t<\infty,
$$

where $x \geqq 0$ means that each component of $x$ is nonnegative. It is remarked there that this result can be considered as a generalization of a well known theorem of Perron-Frobenius on matrices with nonnegative entries. This theorem states that a constant matrix of this type possesses at least one nonnegative eigenvalue, corresponding to which there is a nonnegative eigenvector. There have been a number of generalizations of the latter result to theorems concerning operators on a Banach space transforming some cone into itself; see [2], [3], [4] and the references there. In view of this fact, the question of the possibility of a similar generalization of the above theorem on differential equations naturally arises. It is the purpose of this note to establish such a generalization.

Let $X$ be a Banach space. The following standard notation and terminology will be adopted. $x \in X$ has the norm $|x|$. A cone $K$ is a subset of $X$ such that $x, y \in K$ implies that $\lambda x+\mu y \in K$ when $\lambda, \mu \geqq 0$. $K$ is called proper if $0 \neq x \in K$ implies $-x \notin K$. When $x$ and $y$ are elements of $X, x \geqq y$ means $x-y \in K$, so that in particular $x \geqq 0$ is equivalent to $x \in K$. An operator $A$ on $X$ is said to be nonnegative $(A \geqq 0)$ if $A x \geqq 0$ whenever $x \geqq 0$. A nonempty set of the form $H=\{x: x \in K, f(x)=$ 1) where $f$ is in the dual space $X^{*}$ of $X$, is called a cross-section of the cone $K$.

By the derivative of a function $x(t)$ of the real variable $t$, with values in $X$, is to be understood (except in $\S 6$ ) the limit in the strong topology on $X$, as $h \rightarrow 0$, of the difference quotient $(x(t+h)-x(t)) / h$.

Theorem 1. Let $X$ be a Banach space and $K$ a closed, convex cone in $X$ possessing a wealely compact cross-section $H$. For every fixed $t$, $0 \leqq t<\infty$, let $A(t)$ be a nonnegative, bounded linear operator on $X$ and let $A(t)$ be strongly continuous on $0 \leqq t<\infty$. Then the differential

Received February 27, 1961. 
equation (1) has at least one solution $x=x(t) \not \equiv 0$ satisfying (2).

As in the finite dimensional case, the Theorem 1 has an analogue: for difference equations:

Theorem 2. Let $X, K, H$ be as in Theorem 1. Let $A=A(m)$ be a bounded operator on $X$ defined and nonnegative for each positive integer $m$. Suppose also that $I-A(m)$, where $I$ is the identity operator, has a bounded, nonnegative inverse for $1 \leqq m<\infty$. Then the linear difference equation

$$
\Delta x(m)=-A(m) x(m)
$$

has at least one nontrivial solution satisfying

$$
x(m) \geqq 0, \quad-\Delta x(m) \geqq 0 \text { for } 1 \leqq m<\infty
$$

The proof is similar to that of the theorem for differential equations and will not be given. In connection with the condition that $I-A(m)$, have a bounded, nonnegative inverse, see $\S 5$ below.

2. Proof of Theorem 1. Let $f \in X^{*}$ define a weakly compact crosssection $H$ of $K$, then $f(x)>0$ for all nonzero $x$ in $K$. This is seen in the following way. The set $L$ of elements in $K$ not belonging to the kernel of $f$ projects onto $H$ by the map $P: x \rightarrow x / f(x)$. Assume that there is an $x_{1} \in K, x_{1} \neq 0$, and $f\left(x_{1}\right)=0$. An elementary argument shows that on any line segment connecting $x_{1}$ to $H$ there is a half-open interval, contained in $L$ and having an endpoint $x_{0} \notin L$. It is easy to see that the image under $P$ of such a half-open interval would be unbounded. This contradicts the fact that $H$ is weakly compact, hence bounded.

By the principle of uniform boundedness, $\|A(t)\|$ is bounded on compact $t$-intervals. Let $x=x(t)$ be a solution of (1). The proof will make use of the Gronwall inequality

$$
|x(t)| \leqq|x(s)| e^{M T} \text { for } 0 \leqq s, t \leqq T
$$

where $M$ is a bound for $\|A(t)\|$ on $[0, T]$. This follows from

$$
|x(t)| \leqq|x(s)|+1 \int_{s}^{t}\|A(u)\| \cdot|x(u)| d u \mid .
$$

If $x(t)$ is a solution of (1) and if $x(s) \in K$ for some $s>0$, then $x(t) \in K$, in fact $x(t) \geqq x(s)$, for $0 \leqq t \leqq s$. This is so because $x(t)$ is the limit (in the strong topology) of the sequence of successive approximations defined inductively as follows: $x_{0}(t) \equiv x(s)$ and

$$
x_{n}(t)=x(s)+\int_{t}^{s} A(u) x_{n-1}(u) d u \text { if } n>1 .
$$


Clearly for each $n, x_{n}(t) \geqq x_{n}(s)$ for $0 \leqq t \leqq s$. Since $K$ is closed, this implies $x(t) \geqq x(s)$ for $0 \leqq t \leqq s$.

From the last remark, and the fact that $f(x)>0$ for $0 \neq x \in K$, it follows that for each positive integer $n$, there exists a solution $x=$ $y(t)$ of (1) such that

$$
y(t) \geqq 0 \text { for } 0 \leqq t \leqq n \text { and } y(0) \in H .
$$

Consider the set of all solutions of (1) satisfying (4) for a given $n$, and let $E_{n}$ be the set of their initial values. Thus the $E_{n}$, for $n=1,2, \cdots$ form a nonincreasing sequence of nonempty subsets of $H$. Because of (3), solutions of (1) depend continuously on initial conditions on any finite interval. It follows that each $E_{n}$ is closed. Since the $E_{n}$ are clearly convex, they are weakly closed by Mazur's theorem. Hence the weak compactness of $H$ implies that there must be a point common to all of the $E_{n}$. Any solution of (1) whose initial value is such a point satisfies (2).

3. Cones with weakly compact cross-sections. In most of the well known examples of a Banach space $X$ with a naturally distinguished cone $K$, the cone is generating, i.e., $X=K-K$. Relevant to this fact is the following

(i) A necessary condition that a cone $K$ generating a Banach space $X$ have a weakly compact cross-section is that $X$ be reflexive.

The proposition is an easy consequence of the following

Lemma. Let $K$ be a generating cone in a Banach space $X$, and let $K$ have a weakly compact cross-section $H$. Then there exists a constant $M$ such that every $z \in X$ of norm 1 has a decomposition $z=$ $x-y$ with $x, y \in K$ and $|x|,|y|<M$.

If $K$ is a cone in $X, K^{*}$ below denotes the (dual) cone in $X^{*}$ consisting of elements $f \in X^{*}$ satisfying $f(x) \geqq 0$ for all $x \in K$.

Proof of the lemma. Let $f \in K^{*}$ determine the cross-section $H$. A new norm will be defined on $X$ by

$$
\|z\|=\inf \{f(x)+f(y): z=x-y ; x, y \in K\} ;
$$

in particular, $\|z\|=f(z)$ if $z \in K$. This device is employed by Schaefer [4], p. 1013, and he shows that $X$ is a Banach space with respect to this norm when the cross-section determined by $f$ is weakly compact. Let $S_{1}=\{x: x \in X,|x| \leqq 1\}$ and let $S_{2}=\{x: x \in X,\|x\| \leqq 1\}$. A short computation shows the existence of a positive constant $k$ such that $k S_{2} \subset S_{1}$. It follows from the open mapping principle that the two norms define equivalent topologies. It suffices therefore to prove the lemma for $X$ 
with the new norm.

It is easy to see, from the definition of $\|\cdots\|$, that there exist, for any $z \in X$, elements $x, y \in K$ such that $z=x-y$ and $\|x-y\| \geqq$ $\|x+y\|-1$. Then since $\|x\| \leqq 1 / 2\|x-y\|+1 / 2\|x+y\|$, it follows that if $\|z\|=1$, then $\|x\| \leqq 3 / 2$ and similarly $\|y\| \leqq 3 / 2$.

Proof of (i). From the lemma, it follows that if $\left\{z_{n}\right\}$ is a generalized sequence with $\left|z_{n}\right| \leqq 1$, then there exist bounded sequences $\left\{x_{n}\right\}$ and $\left\{y_{n}\right\}$ with $x_{n}, y_{n} \in K$ and such that for each $n, z_{n}=x_{n}-y_{n}$. Bounded generalized sequences of elements in $K$ have weakly convergent subsequences, hence so has the sequence $\left\{z_{n}\right\}$. Thus the unit sphere $|z| \leqq 1$ in $X$ is weakly compact and so, $X$ is reflexive and (i) is proved.

[Added November 1961. A more direct proof of (i) follows from an observation of H. H. Corson, ("The weak topology of a Banach space," Transactions of the American Mathematical Society, 101 (1961), 1-15), namely a Banach space $X$ is weakly $\sigma$-compact (i.e., a denumerable union of weakly compact sets) if and only if it is reflexive. It is not hard to see that this equivalence remains valid if " $X$ is weakly $\sigma$-compact" is replaced by " $X$ is generated by a weakly compact subset $E$," for if the latter is the case then

$$
X=\bigcup_{n=1}^{\infty}\left\{x: x=a_{1} x_{1}+\cdots a_{n} x_{n},\left|a_{i}\right| \leqq n, x_{i} \in E, i=1, \cdots, n\right\}
$$

is weakly $\sigma$-compact. The assertion (i) is now immediate since a bounded cross-section of a generating cone in a Banach space generates the space.]

If $X$ is reflexive, then a necessary and sufficient condition that a closed cone $K$ in $X$ have a weakly compact cross-section is that $K^{*}$ have an interior element. In fact these two properties for cones in a reflexive Banach space are dual. More generally, one has

(ii) If $X$ is any Banach space with a cone $K$, then $K\left(\right.$ resp. $\left.K^{*}\right)$ has an interior element if and only if $K^{*}$ has a weak* compact crosssection (resp. $K$ has a bounded cross-section).

For a proof of the two nonparenthetical assertions, see [2]. The other assertions, those involving the parenthesis, are contained in the first two but are quite easily proved independently.

4. Special form of Theorem 1. An analogue of the theorem of Perron-Frobenius is the following:

Let $X, K, H$ be as in Theorem $1, A$ a bounded nonnegative"operator. Then $A$ has an eigenvalue $\lambda \geqq 0$ and a corresponding eigenvector $x_{0} \geqq 0$.

This is contained in a stronger theorem of Schaefer [4], pp. 10131014. A very simple proof results from an application of Tychonoff's fixed point theorem to the map $P A$ restricted to $H$, where $P x=x / f(x)$ 
as in $\S 2$ above; cf. [3] for a corresponding proof in the finite-dimensional case.

This analogue of the Perron-Frobenius theorem combined with some arguments of [3] give the following:

Let $X, K, H$ be an in Theorem 1. Let $\Gamma=\{A\}$ be a collection of commutative, bounded, nonnegative operators. Then there is an element $x_{0} \neq 0$ of $K$ which is a common eigenvector of every $A \in \Gamma$ belonging to an eigenvalue $\lambda=\lambda_{A} \geqq 0$.

It can be supposed that $0 \neq x \in K, A \in \Gamma$ imply $A x \neq 0$, for otherwise $\Gamma$ can be replaced by the collection $\{A+I\}$. The arguments in the last half of the proof of Theorem 3.3, [3], can then be used to obtain the desired result. This, in turn, implies a special form of Theorem 1:

Let $X, K, H, A(t)$ be as in Theorem 1. In addition, let $A(t) A(s)=$ $A(s) A(t)$ for $0 \leqq s, t<\infty$. Then (1) has a solution of the form $x=$ $\exp \left(\int_{0}^{t} \lambda(s) d s\right) x_{0}$, where $\lambda(t) \geqq 0$ is continuous for $t \geqq 0$ and $0 \neq x_{0} \geqq 0$.

5. Remark on Theorem 2. The hypothesis of Theorem 2 requires that the operator $I-A(m)$ have a bounded, nonnegative inverse for each positive $m$. Obviously, in order that this condition be satisfied, it suffices for each of the operators $A(m)$ to have a spectral radius $r<1$. It is a consequence of a theorem of Schaefer, [4], pp. 1013-1014, that when $K$ is a generating cone, this sufficient condition is also necessary. Schaefer's theorem implies, in fact, that when $K$ has a weakly compact cross-section and generates $X$, then the spectral radius of any nonnegative operator $A$ belongs to the point spectrum of $A$. (Schaefer's assumption that the cone be "normal" becomes redundant here since the norm generating the topology in $X$ can be altered so as to satisfy $\|x+y\|=$ $\|x\|+\|y\|$ for $x, y \in K$; see Schaefer's proof or lemma in $\S 3$ above.)

6. The dual of Theorem 1. In this section the differential equation

$$
f^{\prime}=-A^{*}(t) f
$$

will be considered, where $A(t)$ is as in Theorem 1 and for each fixed $t$, $0 \leqq t<\infty, A^{*}(t)$ is the adjoint of $A(t)$. A solution of (5) is understood in this case to mean a function $f(t) \in X^{*}$ for $0 \leqq t<\infty$, continuous with respect to the weak* topology, and possessing a weak* derivative satisfying (5). Theorem 1 has the following dual.

Theorem 3. Let $X$ be a Banach space, and let $K$ be a closed cone in $X$ possessing an interior point. Let $A=A(t)$ be as in Theorem 1. Then the differential equation (5) has at least one solution $f=f(t) \not \equiv 0$ satisfying $f(t) \in K^{*}$ for $0 \leqq t<\infty$. 
Proof. Consider the differential equation

$$
x^{\prime}=A(t) x
$$

adjoint to (5). For any point $x_{0} \in X$ there is a uniquely determined, strongly differentiable solution of (6) which takes the value $x_{0}$ when $t=0$. Let $x=x\left(t, x_{0}\right)$ denote this function. It follows from the Gronwall inequality (3) that solutions of (6) depend continuously on initial conditions. Hence for each fixed $t, 0 \leqq t<\infty$, the mapping $x_{0} \rightarrow x\left(t, x_{0}\right)$ is a continuous linear mapping of $X$ onto itself. Let $U=U(t)$ denote this bounded linear operator. Since solutions of (6) are continuous in the norm topology it follows that $U(t)$ is strongly continuous as a function of $t$. Clearly for each fixed $t, U^{-1}(t)$ exists, and by the Gronwall inequality (3), it too is a bounded linear operator for each fixed $t \geqq 0$. Further $U^{-1}(t)$ is a strongly continuous function of $t$. Since $U(t)$ is. strongly differentiable, $U^{-1}(t)$ is as well and $\left[U^{-1}(t)\right]^{\prime}=-U^{-1}(t) A(t)$.

Let $V(t)$, for each $t, 0 \leqq t<\infty$, be the operator on $X^{*}$ which is the adjoint of $U^{-1}(t)$. Since $U^{-1}(t)$ is continuous and differentiable in the strong operator topology, the function $f(t)=V(t) f_{0}$ is continuous and differentiable with respect to the weak* topology on $X^{*}$ for each $f_{0} \in X^{*}$. The function $f(t)=V(t) f_{0}$ is a solution of (5).

Since $K$ has an interior point, $K^{*}$ has a weak* compact cross-section $H^{*}$. Let $E_{n}^{*} \subset H^{*}$ be defined as follows:

$$
E_{n}^{*}=\left\{f_{0}: f_{0} \in H^{*}, V(t) f_{0} \in K^{*} \text { for } 0 \leqq t \leqq n\right\} .
$$

Since $V(t)$ is, for each $t$, the adjoint of a bounded linear operator on $X$, it follows that $V(t)$ is for each $t$ a continuous operator in the weak* topology on $X^{*}$, and hence that each $E_{n}^{*}$ is weak* closed. The $E_{n}^{*}$ clearly form a nonincreasing sequence of sets. It remains to show that each $E_{n}^{*}$ is nonempty. A successive approximation argument similar to that used in the proof of Theorem 1 shows that if $x(t)$ is any solution of (6) and if $x(s) \in K$, for some $s \geqq 0$, then $x(t) \in K$ for $s \leqq t<\infty$. In other words $U(t) U^{-1}(s)$ is a nonnegative operator for $t \geqq s$. By the definition of $V(t), \quad V(t) V^{-1}(n)=\left[U^{-1}(t)\right]^{*}[U(n)]^{*}=\left[U(n) U^{-1}(t)\right]^{*}$. It follows that when $0 \leqq t \leqq n, V(t) V^{-1}(n)$ maps $K^{*}$ into $K^{*}$. Let $g \in K$ and let $f_{0}=V^{-1}(n) g$, then $V(t) f_{0}=V(t) V^{-1}(n) g \in K^{*}$ for $0 \leqq t \leqq n$. Thus, the sets $E_{n}^{*}$ are nonempty for $n=1,2, \cdots$. Since $H^{*}$ is weak* compact, there is an $f \in H^{*}$ with $V(t) f \in K^{*}$ for $0 \leqq t<\infty$.

\section{REFERENCES}

1. P. Hartman and A. Wintner, Linear differential and difference equations with monotone solutions, Amer. J. Math., 75 (1953), 731-743.

2. S. Karlin, Positive operators, J. Math and Mech, 8 (1959), 907-938. 
3. M. G. Krein and M. A. Rutman, Linear operators leaving invariant a cone in a Banach space, Uspehi Matem. Nauk (1948), 3-95 (Amer. Math. Soc. Trans. No. 26).

4. H. Schaefer, Some spectral properties of positive linear operators, Pacific J. Math., 10 (1960), 1009-1019.

THE JoHNS HoPKINS UNIVERSITY 



\section{PACIFIC JOURNAL OF MATHEMATICS}

\section{EDITORS}

Ralph S. Phillips

Stanford University

Stanford, California

M. G. Arsove

University of Washington

Seattle 5, Washington
A. L. Whiteman

University of Southern Californla

Los Angeles 7, California

Lowell J. Paige

University of California

Los Angeles 24, California

\section{ASSOCIATE EDITORS}

E. F. BECKENBACH

D. DERRY

H. L. ROYDEN

E. G. STRAUS

T. M. CHERRY

M. OHTSUKA

E. SPANIER

F. WOLF

\section{SUPPORTING INSTITUTIONS}

UNIVERSITY OF BRITISH COLUMBIA

STANFORD UNIVERSITY

CALIFORNIA INSTITUTE OF TECHNOLOGY

UNIVERSITY OF CALIFORNIA

MONTANA STATE UNIVERSITY

UNIVERSITY OF TOKYO

UNIVERSITY OF UTAH

UNIVERSITY OF NEVADA

NEW MEXICO STATE UNIVERSITY

OREGON STATE UNIVERSITY

UNIVERSITY OF OREGON

OSAKA UNIVERSITY

WASHINGTON STATE UNIVERSITY

UNIVERSITY OF WASHINGTON

UNIVERSITY OF SOUTHERN CALIFORNIA

AMERICAN MATHEMATICAL SOCIETY CALIFORNIA RESEARCH CORPORATION SPACE TECHNOLOGY LABORATORIES NAVAL ORDNANCE TEST STATION 


\section{Pacific Journal of Mathematics}

\section{Vol. 12, No. $1 \quad$ January, 1962}

Jonathan L. Alperin, Groups with finitely many automorphisms $\ldots \ldots \ldots \ldots \ldots \ldots \ldots \ldots$

Martin Arthur Arkowitz, The generalized Whitehead product ................ 7

John D. Baum, Instability and asymptoticity in toplogical dynamics . . . . . . . . . . 25

William Aaron Beyer, Hausdorff dimension of level sets of some Rademacher series .... $\quad 35$

Frank Herbert Brownell, III, A note on Cook's wave-matrix theorem . . . . . . . . . . . . . 47

Gulbank D. Chakerian, An inequality for closed space curves ................. 53

Inge Futtrup Christensen, Some further extensions of a theorem of Marcinkiewicz ....... 59

Charles Vernon Coffman, Linear differential equations on cones in Banach spaces . . . . . 69

Eckford Cohen, Arithmetical notes. III. Certain equally distributed sets of integers . . . . . 77

John Irving Derr and Angus E. Taylor, Operators of meromorphic type with multiple poles

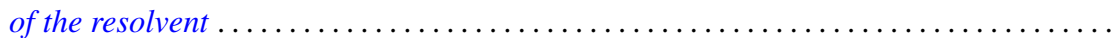

Jacob Feldman, On measurability of stochastic processes in products space .............

Robert S. Freeman, Closed extensions of the Laplace operator determined by a general class of boundary conditions, for unbounded regions ......................

Robert E. Fullerton, Geometric structure of absolute basis systems in a linear topological

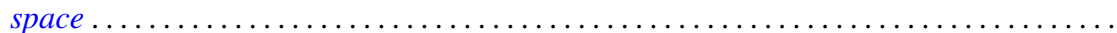

Dieter Gaier, On conformal mapping of nearly circular regions

Andrew Mattei Gleason and Hassler Whitney, The extension of linear functionals defined

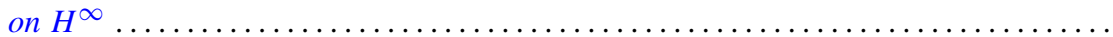

Seymour Goldberg, Closed linear operators and associated continuous linear

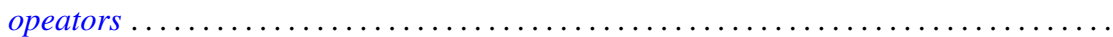

Basil Gordon, Aviezri Siegmund Fraenkel and Ernst Gabor Straus, On the determination of sets by the sets of sums of a certain order

Branko Grünbaum, The dimension of intersections of convex sets. .

Paul Daniel Hill, On the number of pure subgroups

Robert Peter Holten, Generalized Goursat problem . .

Alfred Horn, Eigenvalues of sums of Hermitian matrices ...........

Henry C. Howard, Oscillation and nonoscillation criteria for

$$
y^{\prime \prime}(x)+f(y(x)) p(x)=0
$$

Taqdir Husain, $S$-spaces and the open mapping theorem ...

Richard Eugene Isaac, Markov processes and unique stationary probability measures ...

John Rolfe Isbell, Supercomplete spaces ....................

John Rolfe Isbell, On finite-dimensional uniform spaces. II .........

N. Jacobson, A note on automorphisms of Lie algebras ..............

Antoni A. Kosinski, A theorem on families of acyclic sets and its applications

Marvin David Marcus and H. Minc, The invariance of symmetric functions of singular values...

Ralph David McWilliams, A note on weak sequential convergence.

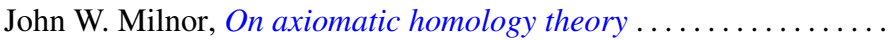

Victor Julius Mizel and Malempati Madhusudana Rao, Nonsymmetric projections in

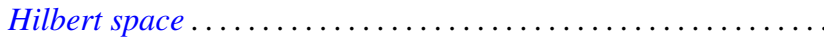

Calvin Cooper Moore, On the Frobenius reciprocity theorem for locally compact

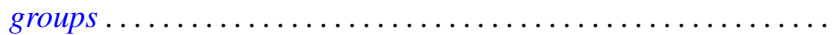

Donald J. Newman, The Gibbs phenomenon for Hausdorff means . 